\title{
Prevalence and antibiotic susceptibility pattern of multidrug resistant gram negative bacilli in lower respiratory tract Infections in a tertiary care hospital of Tripura
}

\author{
Anup Saha ${ }^{1}$, Jayanta Debnath ${ }^{2, *}$, Pradip Kumar Das ${ }^{3}$, Niladri Sekhar Das ${ }^{4}$, Pranjal Tripathi ${ }^{5}$ \\ ${ }^{\mathbf{1}}$ Assistant Professor, ${ }^{\mathbf{2}}$ Associate Professor, ${ }^{3}$ Professor and Head, ${ }^{4}$ Tutor, ${ }^{5}$ MBBS Student, Dept. of Microbiology, Tripura Medical \\ College \& Dr. BR Ambedkar Memorial Teaching Hospital, Tripura, India
}

*Corresponding Author: Jayanta Debnath

Email: drjdebnath@gmail.com

\begin{abstract}
Introduction: Lower respiratory tract infections with multidrug resistant gram negative bacteria are hard to treat and cause high morbidity and mortality. This study gives an account of isolation of multidrug resistant bacterial pathogens causing Lower respiratory tract infections and their antibiogram, in this geographical area of North East India.

Materials and Methods: A total of 500 samples were collected and processed following the standard protocol. Antibiotic susceptibility test were performed by disc diffusion method, from which multidrug resistant strains were detected as per the criteria.

Results: Out of 190 Gram Negative Bacilli isolated, 76 (40\%) strains were confirmed to be multidrug resistant. Klebsiella pneumoniae (45.61\%) was the predominant multidrug resistant Gram Negative Bacilli isolated, followed by Pseudomonas aeruginosa (36.48\%), Acinetobacter spp (33.33\%) and Citrobacter spp (28.57\%). The multidrug resistant strains exhibited considerable susceptibility to Amikacin (65.78\%), Gentamicin (68.42\%) and Levofloxacin (68.42\%). The Carbapenems, Betalactams and Beta-lactamase inhibitor combinations exhibited significant resistance in the present study.

Conclusion: Klebsiella pneumoniae and Pseudomonas aeruginosa can be stated as the major causative agents of LRTI in this region. The present data represents the effective antibiotic agents against the MDR pathogens causing LRTI to be Amikacin, Gentamicin and Levofloxacin. Since none of the MDR strains attained a significant level of sensitivity against the tested antibiotics, specific treatment in our centre need to be decided based upon the antibiotic sensitivity profile of the isolate.
\end{abstract}

Keywords: Lower respiratory tract infections, Gram negative bacilli, Multidrug resistant strains, Antibiotic susceptibility.

\section{Introduction}

Lower respiratory tract infections (LRTI) is a broad description of a group of disease entities, encompassing acute bronchitis, pneumonia and exacerbation of chronic lung disease. ${ }^{1}$ It is one of the leading causes of the morbidity and mortality in developing countries due to the problems associated with the identification of the etiological agents and administration of appropriate treatment. ${ }^{2}$ In recent decades, the increasing prevalence of infections due to multidrug-resistant (MDR) gramnegative bacilli constitutes a serious threat to public health due to slow pace of development of new antimicrobial agents offering limited treatment options. MDR gram-negative bacilli causing LRTI are associated with increased mortality and prolonged hospitalization, which results in a significant burden on healthcare system. ${ }^{3}$ MDR organisms may be associated with either symptomatic respiratory illness or asymptomatic carriage. Differentiating colonization from infections can be difficult and requires clinical correlation. The present study was undertaken to determine the prevalence of multidrug resistant gram negative bacilli causing lower respiratory tract infections and analyze the treatment options available from the antibiotic susceptibility pattern in this geographical area of North East India.

\section{Materials and Methods}

The study was conducted on approval from Institutional Ethical Committee, for duration of one year from January 2016 to December 2016 at Dr. BR Ambedkar Memorial Teaching Hospital, a tertiary care referral institution of the state of Tripura. A total of 500 lower respiratory tract samples including sputum and Endotracheal (ET) aspirates were collected from both out patients and admitted patients attending various departments.

The quality of sputum samples were assessed based on the gram stain findings with 25 or more leucocytes and less than 10 squamous epithelial cells per low power field being considered as good specimen and ideal for processing. ${ }^{4}$ The samples were inoculated onto presterile Blood agar, MacConkey agar and chocolate agar media plates by semi quantitative method. Blood agar and Chocolate agar plates were incubated in 5$10 \% \mathrm{CO}_{2}$ and MacConkey agar plates were incubated aerobically at $37^{\circ} \mathrm{c}$ for $18-24 \mathrm{hrs}$. The identification and characterization of the Gram negative isolates were based on colony morphology, Gram stain findings, motility test and a battery of biochemical tests which included catalase test, sugar fermentation tests, triple sugar iron agar reaction, indole production test, methyl red test, voges proskauer test, citrate utilization test, urease production test, oxidase test. ${ }^{5}$ Antibiotic susceptibility test was performed on the Gram negative 
isolates by modified Kirby Bauer's disc diffusion method following clinical and laboratory standard Institute (CLSI) guidelines. ${ }^{6}$

On analysis of the antibiotic susceptibility pattern, MDR strains of the isolated Gram negative bacilli were detected by abiding to the criteria stating that those which are resistant to three or more antibiotic classes. ${ }^{7}$ For quality control E.coli ATCC 25922, P.aeruginosa ATCC 27853 and K. pneumoniae ATCC 700603 were used.

\section{Results}

During the study period, out of 500 samples processed for culture, 470 samples were sputum and 30 samples were endotracheal aspirates. A total of 200 numbers of positive cultures yielded bacterial pathogens, of which 190 isolates were Gram negative bacilli. Klebsiella pneumoniae $(60 \%)$ was identified as predominant pathogen followed by Pseudomonas aeruginosa (20\%), Acinetobacter spp. (9.47\%), Citrobacter freundii (7.36\%) and Enterobacter spp., Escherichia coli and Edwardsiella spp. of $1.05 \%$ each, as shown in Table 1.

Table 1: Spectrum and proportion of culture isolates of Gram negative bacilli

\begin{tabular}{|l|c|c|c|}
\hline Bacterial isolate & $\begin{array}{c}\text { Number of isolates in } \\
\text { Sputum }\end{array}$ & $\begin{array}{c}\text { Number of isolates in } \\
\text { Endotracheal aspirates }\end{array}$ & $\begin{array}{c}\text { Total Number of } \\
\text { Isolates [N }(\%)]\end{array}$ \\
\hline Klebsiella pneumoniae & 106 & 08 & $114(60 \%)$ \\
\hline Pseudomonas aeruginosa & 30 & 08 & $38(20 \%)$ \\
\hline Acinetobacter spp. & 18 & 00 & $18(9.47 \%)$ \\
\hline Citrobacter freundii & 12 & 02 & $14(7.36 \%)$ \\
\hline Enterobacter spp. & 02 & 00 & $02(1.05 \%)$ \\
\hline Escherichia coli & 02 & 00 & $02(1.05 \%)$ \\
\hline Edwardsiella spp. & 02 & 00 & $02(1.05 \%)$ \\
\hline
\end{tabular}

The pattern of antibiotic susceptibility of the isolated Gram negative bacilli has been depicted in Table 2. It was observed that amikacin, gentamicin and levofloxacin were the most sensitive drugs to most of the isolates, ranging from $71.42 \%$ to $100 \%$, except Pseudomonas aeruginosa which exhibited 52.63\% sensitivity towards amikacin. Comparatively, the isolates showed lower level of sensitivity to Imipenem and piperacillin tazobactum, ranging from as low as $21.05 \%$ to $100 \%$. The widely prescribed cephalosporin group of antibiotics were considerably resistant, of which cefuroxime was highly resistant to the isolates, its sensitivity ranging from 0 to $42.85 \%$, except Enterobacter spp.

Table 2: Antibiotic sensitivity pattern of isolated gram negative bacilli [\%(n)]

\begin{tabular}{|l|c|c|c|c|c|c|c|}
\hline Antibiotics & $\begin{array}{c}\text { Klebsiella } \\
\text { pneumoniae } \\
(\mathbf{n = 1 1 4}\end{array}$ & $\begin{array}{c}\text { Pseudomonas } \\
\text { aeruginosa } \\
(\mathbf{n = 3 8}\end{array}$ & $\begin{array}{c}\text { Acinetobacter } \\
\mathbf{s p p .}(\mathbf{n = 1 8})\end{array}$ & $\begin{array}{c}\text { Citrobacter } \\
\text { freundii }(\mathbf{n = 1 4})\end{array}$ & $\begin{array}{c}\text { Enterobacter } \\
\mathbf{s p p .}(\mathbf{n = 2})\end{array}$ & $\begin{array}{c}\text { Escherichia } \\
\text { coli }(\mathbf{n = 2})\end{array}$ & $\begin{array}{c}\text { Edwardsiella } \\
\text { spp. }(\mathbf{n = 2})\end{array}$ \\
\hline $\begin{array}{l}\text { Piperacillin } \\
\text { Tazobactum }\end{array}$ & $64.91 \%(74)$ & $21.05 \%(08)$ & $66.66 \%(12)$ & $47.14 \%(08)$ & $100 \%(02)$ & $100 \%(02)$ & $0.00 \%(00)$ \\
\hline Amikacin & $91.22 \%$ & $53.63 \%(20)$ & $77.77 \%(14)$ & $85.71 \%(12)$ & $100 \%(02)$ & $100 \%(02)$ & $100 \%(02)$ \\
\hline Gentamicin & $84.21 \%(96)$ & $89.47 \%(34)$ & $88.88 \%(16)$ & $71.42 \%(10)$ & $100 \%(02)$ & $100 \%(02)$ & $100 \%(02)$ \\
\hline Levofloxacin & $82.45 \%(94)$ & $84.21 \%(32)$ & $100 \%(18)$ & $71.42 \%(10)$ & $100 \%(02)$ & $100 \%(02)$ & $100 \%(02)$ \\
\hline Ciprofloxacin & $61.40 \%(70)$ & $57.89 \%(22)$ & $55.55 \%(10)$ & $42.85 \%(06)$ & $0.00 \%(00)$ & $0.00 \%(00)$ & $100 \%(02)$ \\
\hline Cefuroxime & $24.56 \%(28)$ & $31.57 \%(12)$ & $11.11 \%(02)$ & $42.85 \%(06)$ & $100 \%(02)$ & $0.00 \%(00)$ & $0.00 \%(00)$ \\
\hline Ceftriaxone & $63.15 \%(72)$ & $42.10 \%(16)$ & $77.77 \%(14)$ & $57.14 \%(08)$ & $100 \%(02)$ & $0.00 \%(00)$ & $0.00 \%(00)$ \\
\hline Ceftazidime & $50.87 \%(58)$ & $73.68 \%(28)$ & $44.44 \%(08)$ & $85.71 \%(12)$ & $0.00 \%(00)$ & $0.00 \%(00)$ & $0.00 \%(00)$ \\
\hline Cefepime & $54.38 \%$ & $26.31 \%$ & $22.22 \%$ & $57.14 \%(08)$ & $100 \%(02)$ & $100 \%(02)$ & $100 \%(02)$ \\
\hline Imipenem & $75.43 \%$ & $89.47 \%(34)$ & $100 \%(18)$ & $57.14 \%(08)$ & $100 \%(02)$ & $100 \%(02)$ & $100 \%(02)$ \\
\hline
\end{tabular}

MDR strains were detected in $76(40 \%)$ of the isolated Gram negative bacilli as illustrated in Fig. 1.



In our study $36.84 \%$ (28/76) of MDR pathogen causing LRTI were associated with risk factors which are depicted in Fig. 2.

Fig. 1: Prevalence of MDR GNB in LRTIs 




Fig. 2: Risk factors associated with MDR pathogen causing LRTI

MDR strains were detected in Klebsiella pneumoniae (45.61\%), Pseudomonas aeruginosa (36.48\%), Acinetobacter spp (33.33\%) and Citrobacter freundii (28.57\%) as shown in Fig. 3.



Fig. 3: Organism wise frequency of MDR GNB [\%(n)]

The overall antibiotic susceptibility pattern of the MDR Gram negative bacilli strains has been depicted in Table 3. It was observed that, the most resistant antibiotic was cefuroxime $(89.47 \%)$, followed by ciprofloxacin $(84.21 \%)$. Most of the strains exhibited considerable sensitivity to gentamicin (68.42\%), levofloxacin (68.42\%) and amikacin (65.78\%). More than $60 \%$ of the MDR strains were resistant to the third and fourth generation cephalosporins. On the contrary, the MDR strains exhibited $44.73 \%$ and $78.94 \%$ resistance to imipenem and piperacillin tazobactum respectively.

Table 3: Antibiotic susceptibility pattern of MDR Gram negative bacilli ( $N=76$ )

\begin{tabular}{|l|c|c|}
\hline Antibiotics & Sensitive[\%(n)] & Resistant[\%(n)] \\
\hline Piperacillin/Tazobactum & $21.05 \%(16)$ & $78.94 \%(60)$ \\
\hline Amikacin & $65.78 \%(50)$ & $34.21 \%(26)$ \\
\hline Gentamicin & $68.42 \%(52)$ & $31.57 \%(24)$ \\
\hline Levofloxacin & $68.42 \%(52)$ & $31.57 \%(24)$ \\
\hline Ciprofloxacin & $15.78 \%(12)$ & $84.21 \%(64)$ \\
\hline Cefuroxime & $10.52 \%(8)$ & $89.47 \%(68)$ \\
\hline Ceftriaxone & $34.21 \%(26)$ & $65.78 \%(50)$ \\
\hline Ceftazidime & $39.47 \%(30)$ & $60.52 \%(46)$ \\
\hline Cefepime & $28.94 \%(22)$ & $71.05 \%(54)$ \\
\hline Imipenem & $55.26 \%(42)$ & $44.73 \%(34)$ \\
\hline
\end{tabular}

On analysis of antibiotic resistance pattern of the detected MDR strains of the Gram negative bacilli, it was observed that levofloxacin, gentamicin and amikacin are least resistant to all the MDR strains whereas piperacillin-tazobactum, ciprofloxacin and cephalosporin group of antibiotics are highly resistant to them.

\section{Discussion}

LRTIs are among the most common infectious diseases worldwide, of which the MDR pathogens are associated with high morbidity and mortality for all age groups. The World Health Organization has identified antimicrobial resistance as one of the three greatest threats to human health. ${ }^{8}$ With the recent knowledge 
about the spectrum of pathogens and antibiotic susceptibility pattern, an empirical regimen can be formulated to administer in patients with an objective to reduce the risk of mortality and potential complications.

In the present study, Gram negative bacilli were isolated from both Sputum and Endotracheal aspirates, the isolation rate being 38\%. El Askary et al also reported $44.1 \%$ isolation of Gram negative bacilli from patients with LRTI. ${ }^{9}$

Klebsiella pneumoniae $(60 \%)$ was the predominant isolated Gram negative bacilli followed by Pseudomonas aeruginosa (20\%), Acinetobacter spp. (9.47\%) and Citrobacter freundii (7.36\%). Klebsiella pneumoniae was the predominant isolated pathogen reportedin other studies by Biswas $\mathrm{P}$ et al and Tripathi $\mathrm{P}$ et al. ${ }^{10,11}$ Mannur $\mathrm{S}$ et al stated Pseudomonas aeruginosa to be the predominant pathogen in LRTI. ${ }^{12}$ This variation in spectrum of organisms may be attributed to different geographical and clinical conditions with associated risk factors.

In the present study, MDR strains were detected in $40 \%$ of the Gram negative isolates which is in concordance with observation stated by Vishwanath et al(37\%). ${ }^{1}$ But Gagneja et al reported $83 \%$ of MDR Gram negative bacilli isolated from lower respiratory tract of ICU patients. ${ }^{13}$ On the contrary, Deotale et al reported $26.9 \%$ of the isolated Gram negative bacilli were MDR. ${ }^{14}$ Among the isolated MDR Gram negative bacilli, we observed that $45.61 \%$ were Klebsiella pneumoniae followed by Pseudomonas aeruginosa (36.48\%), Acinetobacter spp (33.33\%) and Citrobacter freundii $(28.57 \%)$. El Askary et al also reported similar observation, stating $45.5 \%$ of Klebsiella pneumoniae and $42.2 \%$ of Pseudomonas aeruginosa to be MDR. ${ }^{9}$ In contrast to that Pseudomonas aeruginosa was the predominant MDR strains reported by Goel et al. ${ }^{15}$

In respective of associated risk factors, the present study indicates that patients with multiple comorbidities (14.47\%) are more prone to develop multidrug resistant bacterial infection. On the contrary, Mannur S et al stated Diabetes mellitus $(8.5 \%)$ as predominant risk factor for LRTI. ${ }^{12}$ This variation could be explained by immune status of the patient as well as injudicious use of antibiotics which determine the bacterial drug resistance. Besides this other risk factors which are found in this study are diabetes mellitus (11.84\%), Smoking (5.26\%), COPD (2.63\%), Alcohol and Hypertension $1.31 \%$ each.

In most of the previous studies, the effective treatment choice of MDR Gram negative bacilli were reported to be carbapenems, beta lactamase inhibitors, aminoglycosides and piperacillin tazobactum. We observed a different scenario in the present study. On analysis of the antibiotic sensitivity pattern, it was observed that amikacin $(65.78 \%)$, gentamicin $(68.42 \%)$ and levofloxacin $(68.42 \%)$ were the antibiotics which had higher sensitivity to the MDR pathogens. But comparatively, only $55.26 \%$ MDR isolates were sensitive to imipenem. The MDR isolates exhibited higher level of resistance against cefuroxime $(89.47 \%)$, Ciprofloxacin $(84.21 \%)$ and piperacillin tazobactum $(78.94 \%)$. Viswanath et al reported that $69.7 \%$ of the MDR isolates were sensitive to amikacin, $38.9 \%$ to gentamicin, $27.4 \%$ to ciprofloxacin and $48.6 \%$ to piperacillin tazobactum. ${ }^{1}$ El Askary et al reported that $59.9 \%$ of the Gram negative bacilli isolated from LRTI were sensitive to imipenem, $36.9 \%$ to amikacin, $28.9 \%$ to gentamicin and $37.8 \%$ to ciprofloxacin. ${ }^{9}$

We observed that, the MDR isolates failed to attain a level of sensitivity of more than $70 \%$ against the tested antibiotics. This indicates that specific antibiotic therapy against MDR pathogens causing LRTI in our centre need to be decided based upon the antibiotic sensitivity profile of the isolate. However, in the period while waiting for the culture and sensitivity report, aminoglycosides and levofloxacin could be the drug of choice to initiate treatment of suspected cases of LRTI in our location.

\section{Conclusion}

Klebsiella pneumoniae and pseudomonas aeruginosa can be stated as the major causative agents of LRTI in this region. The present data represents the effective antibiotic agents against the MDR pathogens causing LRTI to be amikacin, gentamicin and levofloxacin. Since none of the MDR strains attained a significant level of sensitivity against the tested antibiotics, treatment in our centre need to be decided based upon the antibiotic sensitivity profile of the isolate. The cause of considerable prevalence of MDR strains need to be explored in this region by further studies.

\section{Conflict of Interest: Nil}

\section{References}

1. Vishwanath S, Chawla K, Gopinathan A. Multidrug resistant Gram negative bacilli in lower respiratory tract infections. Iran J Microbiol. 2013;5:323-327.

2. Khan S, Priti S, Ankit S. Bacteria etiological agents causing lower respiratory tract infections in the western part of Nepal. Ibnosina J Med BS. 2014;6:3-8.

3. Cerceo E, Deitelzweig SB, Sherman BM, Amin AM. Multidrug resistant gram negative bacterial infections in hospital settings: overview, implications for clinical practice and emerging treatment options. Microbial Drug Resistance. 2016;22:412-431.

4. Lentino JR, Lucks DA. Nonvalue of sputum culture in the management of lower respiratory tract infections. J Clin Microbiol. 1987;25:758-762.

5. Bailey \& Scott's Diagnostic Microbiology, Betty AF, Daniel FS, Alice SW, eds. 12th edn, Overview of Bacterial Identification Methods and strategies. Mosby Elsevier, Philadelphia, USA, 2007. 216-247.

6. Clinical and Laboratory Standards Institute (CLSI). Performance standards for antimicrobial susceptibility testing: Twenty fourth informational supplement, M100S24, January. 2014;34:44 -93. 
7. Magiorakos AP, Srinivasan A, Carey RB, Carmeli Y, Falagas ME, Giske CG, et al. Multidrug resistant, extensively drug resistant and pandrug resistant bacteria: an international expert proposal for interim standard definitions for acquired resistance. Clin Microbiol Infect. 2012;18:268-281.

8. Gilbert DN, Guiodos RJ, Boucher HW, Talbot GH, Spelberg B, Edwards Jr JE et al. The 10 X 20 initiative: pursuing a global commitment to develop 10 new antibacterial drugs by 2020. Clin Infect Dis. 2010;50:1081-1083.

9. El Askary SA, Makfled AF, Abdel Aal GA, Ibrahem RA. Prevalence of Aerobic gram negative bacilli in lower respiratory tract infections in Menoufia Governorate, Egypt. Greener J Med Sci. 2016;6:52-62.

10. Biswas P, Tukaram P. Bacterial causes of lower respiratory tract infection in patients attending central referral hospital, Gangtok with reference to antibiotic resistance pattern. J Evol Med Dent Sci. 2013;2:81268135.

11. Tripathi Purti C, Dhote K. Lower Respiratory Tract Infection: Current Etiological Trends and Antibiogram. J Pharm Biomed Sci. 2014;4:249-255.

12. Mannur S, Shubhakara K, Nagaraj ER. Study of risk factors, chest $\mathrm{X}$ - ray findings, aetiological agents and their sensitivity pattern among patients with lower respiratory tract infection. Int J Pharm Bio Sci. 2015;6:336-341.

13. Gagneja D, Goel N, Aggarwal R, Chaudhary U. Changing trend of antimicrobial resistance among gram negative bacilli isolated from lower respiratory tract of ICU patients : A 5 year study. Indian J Crit Care Med. 2011;15:164-167.

14. Deotale VS, Attal R, Narang P. Increase in antibiotic resistance: are bacteria growing with pan resistance?. Int J Cur Res Rev. 2015;7:43-47.

15. Goel N, Chaudhary U, Aggarwal R, Bala K. Antibiotic sensitivity pattern of gram negative bacilli isolated from lower respiratory tract of ventilated patients in intensive care unit. Indian J Crit Care Med. 2009;13:148-151.

How to cite this article: Saha A, Debnath J, Das P K, Das N S, Tripathi P. Prevalence and antibiotic susceptibility pattern of multidrug resistant gram negative bacilli in lower respiratory tract Infections in a tertiary care hospital of Tripura. Indian J Microbiol Res. 2018;5(4):538-542. 